\title{
Investigation for Location Preferences of MIG Household in West Zone of Surat City
}

\author{
Dr. Digant A. Pastagia ${ }^{1}$, Prof. Kevin N. Modi ${ }^{2}$
}

${ }^{1}$ Assistant Professor, CED, Shree Swami Atmanand Saraswati Institute of Technology, Surat, Gujarat, India ${ }^{2}$ Assistant Professor, CED, BMCET, Surat, Gujarat, India

\begin{abstract}
Urbanization growth in India is increasing rapidly and people migrate to the well- developed area of city for various basis. "Residential Location Preferences" refers to, how location of a house of a particular class of people gets determined by their social, economic aspects and also by potential of particular area chosen for residence. Residential location is one of the driving forces of urban dynamics. The residential location choice process has been studied for several decades using different approaches which in general shows that many factors contribute to the choice of a given location as characteristics of city infrastructure, transportation facilities, housing unit, social and environmental amenities, access to jobs, services and other economic opportunities. Urban citizens intend to live surrounding their work locations and city centre. The assessment of these ward locational preferences is done by developing the Weighted Sum Model (WSM). Study represents locational preferences of residence from MIG-1 and MIG -2 categories of residents in west zone of Surat city.
\end{abstract}

Keywords : Residential location Preferences, Housing location, MIG, Neighbourhood, WSM, Ward Potential Value

\section{INTRODUCTION}

In all land uses Residential land use is predominant. These residential areas are divided into various zones depending upon various characteristics such as connectivity, surroundings, facilities and amenities available, distance from work place, etc. Depending upon these characteristics different zones in residential land use observes different scale and pace of development and has different property values. (1) For any family to decide on the buying or renting a house there are many parameters. The most important of all is affordability. (2) Along with this the distance from the work place, availability of amenities and 
facilities also affects the location of housing. For people with different income groups and social background, preferences to the location of house vary. Many a times certain area is occupied by the people of equal income group and various zones in the residential area are formed. Particular residential area often has a particular housing typology and particular spatial arrangement arising out of it. (3) The study area of city can be defined by its population, demographic and economic growth. It classifies the residential growth of particular urban area. The demographic characteristics of the city give the density and trend of growth of population in each ward of zone which will ultimately be useful for making the study of residents' location. Economic characteristics of the area will identify the major activity in the area and its having an influence in residential location. West zone of Surat city is developing zone and it has most middle income group residents. West zone also known as Rander zone, is situated on the west side of Surat city. The west zone is considered for present study as it is established mainly as residential land use.

\section{STUDY AREA PROFILE}

Surat is a city located on the western part of India in the state of Gujarat. It is one of the most dynamic city of India with one of the fastest growth rate due to immigration from various part of Gujarat and other states of India. West zone of Surat is a residential area reporting decadal growth rate of $48 \%$ in last decade. (4) Also, West zone is very nearby to CBD and its having good transportation connectivity with surrounding zones. The total study area is $51.27 \mathrm{sq} . \mathrm{km}$.

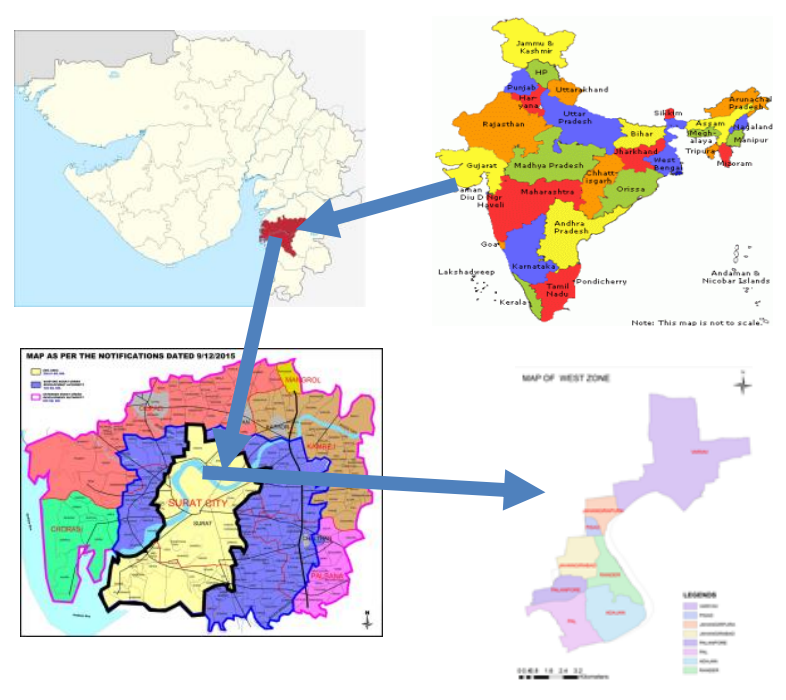

Figure: 1 . Location of study area

West Zone also known as Rander Zone of the Surat city. Which is enclosed by the River Tapti. West Zone of the city is one of the oldest and conjoin to the walled city area. The population growth rates are high, indicating the rapid growth of congestion in the residential areas. It is clearly observed from above Table that as far as the decade growth is concerned for the west zone. Adajan, pal, Palanpur has a highest growth during decade 1991-2001 where in jahangirabad, pal has reported highest growth rate during 2001-2011.

The west zone in particular has shown rise in population. Most of this zone prefer for intercity migration. There are eight wards in west zone. The most developed ward is adajan. The area of adajan is 
near to CBD area and in pal and Palanpur is the most developing area in west zone. Reducing population growth rate in specific wards indicate the substantial change in the land use i.e residential to commercial conversion of building use allowing more people to work in these particular areas.

\section{HOUSING CLASSIFICATION BASED ON ECONOMIC GROUP}

Housing is also classified based on the socio-economic classes slated to occupy them. Classified as High Income Group (HIG), Middle Income Group (MIG), Lower Income Group (LIG) and Economically Weaker Sections (EWS) housing typologies. The income slabs for this socio-economic distribution is given and revised by Ministry of Housing and Urban Poverty Alleviation (MoHUPA) and currently stands at the following (5)(India, January 2017):

- EWS - Annual Income up to INR 3 lakh

- LIG - Annual income between 3 - below 6 lakh

- MIG 1- Annual income between 6-below 12 lakh

- MIG 2- Annual income between 12-below 18 lakh

\section{SELECTION OF STUDY PARAMETER}

The study area in the present work is West Zone of Surat City and it is taken up to understand the residential location choice behavior. The study analysis is based on Data Collected through Home Interviews conducted in Eight wards namely - Rander, Adajan, Jahangirpura, Jahangirabad, Pal, Palanpur, Pisad, Variav. This study analysis focuses on the following issues:
- Housing Attributes Income

- Based Preferences Locational

- Ranking Analysis

Demographic profile, Infrastructural facilities and the travel behavior are equally important in understanding the housing activities in the study areas. (9) Location details, it contains the information regarding location of the particular dwelling unit and building form details, floor area, ownership and the Years of occupancy of particular dwellers. (6) SocioEconomic details, in which family details, Economic details and Work location details is covered. The main reasons are divided in five factors namely environmental factors, Infrastructural factors, Coast factor, Amenities factor and Work location factor each factor are divided in to some sub factors. (7) The field interviews were carried out by the data collection at home.

1. Environment Factor

- Pollution

- Gardens

- Open Spaces

2. Infrastructure factor

- Water supply

- Sewerage connection

- Solid waste collection

- Storm water connection

3. Affordability

4. Amenities

- Accessibility

- Health facility 
- Market facility

- Education facility

5. Transportation Connectivity to Work Place

The Average rating was given to different factors for different income groups (8). For example, environmental factor, there sub-factors and each was given different rates by respondents. The average rating was calculated by taking average rating of these three environmental sub-factors for particular group. Similarly, average rating was found out for different factors according to different groups.

To find the residents' ward wise ranking with Multiple Factor Ranking Method the Weights has been assigned to each Income Group is as considered.

Table 4. Residents' Ward Wise Ranking

\begin{tabular}{|c|c|}
\hline Income Group & Weight \\
\hline MIG I & 50 \\
\hline MIG II & 50 \\
\hline
\end{tabular}

Rander ward (16)

Average Preference Rating for Rander Ward
Table 5 Average Preference Rating for Rander Ward

\begin{tabular}{|c|c|c|}
\hline Factors & \multicolumn{2}{|c|}{ Income Group } \\
\hline & MIG I & MIG II \\
\hline Weight & 50 & 50 \\
\hline Environment & 6.47 & 6.53 \\
\hline Infrastructure & 7.6 & 7.71 \\
\hline Cost & 6.73 & 6.68 \\
\hline Amenities & 7.4 & 6.85 \\
\hline Work location & 7.13 & 7.8 \\
\hline
\end{tabular}

The average preference for the different factors in selecting their residence, for MIG group in Rander ward. The average rating is given and preference matrix was prepared Rander ward. This Preference matrix was further analyse and converted into Normalised matrix form to find out the preference score of MIG groups.

Table 6 Normalized matrix with Preference Score for Rander Ward

\begin{tabular}{|c|c|c|c|}
\hline Factors & MIG I & MIG II & $\begin{array}{c}\text { Preference } \\
\text { Score }\end{array}$ \\
\hline Weight & 0.50 & 0.50 & \\
\hline Environment & 0.99 & 1 & 0.99 \\
\hline Infrastructure & 0.98 & 1 & 0.98 \\
\hline Cost & 1 & 0.99 & 0.99 \\
\hline Amenities & 0.92 & 1 & 0.96 \\
\hline $\begin{array}{c}\text { Work } \\
\text { location }\end{array}$ & 0.91 & 1 & 0.95 \\
\hline
\end{tabular}


The Normalised matrix of preference matrix. It is clearly observed that maximum performance score for Rander ward is found 0.99 for the Cost factor and Environment. Whereas infrastructure and Amenities factors performance score was found out 0.98 and 0.96 and Work Locations performance score was found least out of all factors which is 0.95 .

\section{WARD ATTRACTION VALUE}

Ward attraction value of each ward based on performance score are shown in below table. The performance score values were generated by adding each ward performance scores of all factors.

Table 7. Ward attraction Value

\begin{tabular}{|c|c|c|c|c|c|c|c|c|}
\hline Factors & Adajan & Jahangirabad & Jahangirpura & Palanpur & Rander & Pisad & Pal & Variyav \\
\hline Environment & 0.97 & 0.96 & 0.99 & 0.93 & 0.99 & 0.98 & 0.99 & 0.91 \\
\hline infra & 0.98 & 0.98 & 0.99 & 0.99 & 0.98 & 0.98 & 0.99 & 0.98 \\
\hline Cost & 0.94 & 0.97 & 0.87 & 0.92 & 0.99 & 0.90 & 0.92 & 0.99 \\
\hline Amenities & 0.97 & 0.95 & 0.99 & 0.94 & 0.96 & 0.97 & 0.95 & 0.99 \\
\hline Work & 0.91 & 0.93 & 0.94 & 0.91 & 0.95 & 0.98 & 0.98 & 0.92 \\
location & 4.77 & 4.79 & 4.78 & 4.69 & 4.87 & 4.81 & 4.84 & 4.79 \\
\hline WAV & 6 & 4 & 5 & 7 & 1 & 3 & 2 & 4 \\
\hline RANK & 6 & 5 & & & & & & \\
\hline
\end{tabular}

Above table clearly shows that the ward attraction value for Rander ward is highest among all wards. Community aspects of the Rander ward and its Environment and lower cost are the main reason behind the high value of this ward. While Palanpur having a lowest value of ward attraction because of its long distance from CBD area and poor connectivity with other zones. As per ward potential value ranking given to wards. Maps clearly shows the ranking of wards. 


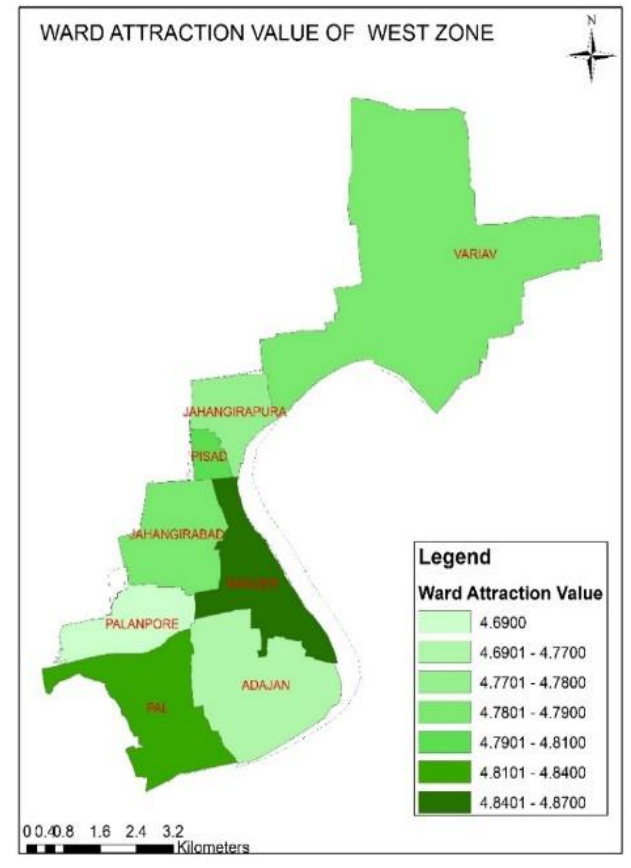

Figure 1. Ward Attraction Value

\section{WARD POTENTIAL VALUE}

The ward Potential value is the value which indicates that which ward is having highest potential to grow in future after considering all the factors. To find WPV the factors are divided in to two groups. And that are (1) Ward Push Factors, (2) Ward Pull factors.

1) Ward Push Factors

The factors which are having positive effects on peoples' choice and attracting the people to choose the particular ward as their residential location The ward push factors considered in study are Environment, Infrastructure factor and Amenities factor

2) Ward Pull factors

It is defined as a negative measurement of combined effect of residential choice factors discouraging the dwellers to decide the location.
The ward pull factors considered in study are Cost and Work location factor.

Now the Ward Potential Value (WpoV) can found out by taking difference of Ward Push factor (W Ps F) and Ward Pull factor (W Pl F)

Table 8. Ward Potential Value

\begin{tabular}{|c|c|c|c|c|}
\hline Ward No. & Ward Name & W Ps V & W Pl V & WpoV \\
\hline 16 & Rander & 2.93 & 1.94 & 0.99 \\
\hline 17 & Adajan & 2.92 & 1.85 & 1.07 \\
\hline 63 & Jahangirabad & 2.89 & 1.9 & 0.99 \\
\hline 64 & Jahangirpura & 2.97 & 1.81 & 1.16 \\
\hline 65 & Pisad & 2.93 & 1.88 & 1.05 \\
\hline 67 & Pal & 2.93 & 1.9 & 1.03 \\
\hline 68 & Palanpor & 2.86 & 1.83 & 1.03 \\
\hline 69 & Variyav & 2.88 & 1.91 & 0.97 \\
\hline
\end{tabular}

It is observed from the above table that Jahangirpura is having a maximum Ward Potential Value, affordable land prices and its excellent road connectivity are the major aspects behind the maximum potential value, Variyav ward is having least $\mathrm{WpoV}$ as this ward is lack of infrastructure facilities and other reason is this ward is fully saturated. This could be the reason behind the low potential value. The map indicates that after Jahangirpura, Adajan ward is having second highest potential value, the implementation of T.P. Schemes and well developed infrastructure are the main aspects behind its potential 


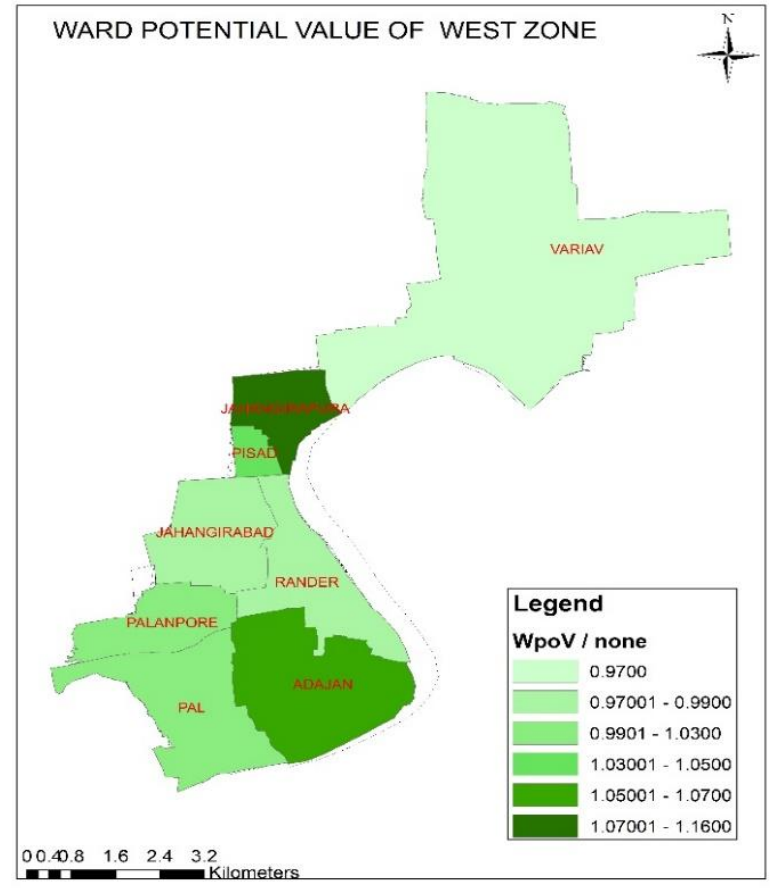

Figure 2 Ward Potential value

\section{FINDINGS AND CONCLUSION}

The west zone of Surat city having $15 \%$ of total city area and accommodating city population. This highly growing area of city having eight different wards namely - Rander, Adajan, Jahangirpura, Jahangirabad, $\mathrm{Pal}$, Palanpur, pisad, variyav and it is rising as residential zone. This upcoming residential zone attracting more people from other parts of the city and looking to this developers more develop their sites and local body, SMC implementing more numbers of T.P schemes. The study shows that this area is having high attraction factor for residential location Preferences of peoples looking to the data analysis following conclusion were drawn. The findings of the present study are concluded below. Ward attraction value based on performance score shows that the Rander ward is highest among all wards. Community aspects of the Rander ward and its Environment and lower cost are the main reason behind the high value of this ward. While Palanpur having a lowest value of ward attraction because of its long distance from CBD area and poor connectivity with other zones

It is observed from the above table that Jahangirpura is having a maximum Ward Potential Value, affordable land prices and its excellent road connectivity are the major aspects behind the maximum potential value, Variyav ward is having least $\mathrm{WpoV}$ as this ward is lack of infrastructure facilities and other reason is this ward is fully saturated. This could be the reason behind the low potential value.

\section{REFERENCES}

[1]. A. P. Verma, Can. J. Phy. 47, 1969, 2519-2524

[2]. O. A. Oleinik, A. S. Kalashnikov and CHZ HOU Yui-Lin, 'The Cauchy problem and boundary problems for equations of the type unsteady filteration' , Izv, Akad, SSSR Ser. Mat. 22, 1958, 667-704.

[3]. F. V. Atkinson and L. A. Peletier, 'Similarity profiles of flows through Porous Medium', Arch. Rational Mech. Anal 42, 1961, 369-379.

[4]. F. V. Atkinson and L. A. Peletier. 'Similarity solutions of the non-linear diffusion equation', Arch. Rational Mech. Anal. 54, 1974, 373-392.

[5]. L.F. Shampine, 'Concentration dependent diffusion', Quart, Appl. Maths. 30, 1973, 441452. 
[6]. L. F. Shampine, 'Concentration dependent diffusion-II singular problems', Quart, Appl. Maths. 31, 1973, 287-452.

[7]. P. Hartman, 'Ordinary differential Equation', John Wiley and sons Inc. New York, 1964.

[8]. G. L. Barenblatt, 'On some unsteady motions of a liquids and a gas in a porous medium', Prinkl, Mat. Mech. 16, 1952, 67-68.

\section{Cite this article as :}

Dr. Digant A. Pastagia, Prof. Kevin N. Modi, "Investigation for Location Preferences of MIG Household in West Zone of Surat City ", International Journal of Scientific Research in Science and Technology (IJSRST), Online ISSN : 2395-602X, Print ISSN : 2395-6011, Volume 6 Issue 1, pp. 564-571, January-February 2019. Available at doi : https://doi.org/10.32628/IJSRST19690

Journal URL : https://ijsrst.com/IJSRST19690 